\title{
PERFORMANCE ANALYSIS FOR THE AF-BASED FREQUENCY DIVISION COOPERATIVE BROADCAST CHANNEL
}

\author{
E. V. Belmega, B. Djeumou and S. Lasaulce \\ Laboratoire des Signaux et Systèmes \\ CNRS - Supélec - Paris 11 \\ 3 rue Joliot Curie, 91192 Gif-sur-Yvette - France
}

\begin{abstract}
This paper considers a system where one transmitter broadcasts a single common message to two receivers. These receivers can cooperate through a bidirectional channel that is assumed to be orthogonal to the downlink channel. For the case where the assumed cooperation protocol is amplify-and-forward we calculated the final equivalent SNR in the MRC output at each receiver for an arbitrary number of cooperation exchanges. The corresponding analytical expressions can then be used for evaluating different performance criteria in order to discuss issues such as: Which receiver should start cooperating first? Is there an optimum number of cooperation exchanges? What is the difference between asymmetric and symmetric cooperations?
\end{abstract}

\section{INTRODUCTION}

In the conventional broadcast channel (BC) introduced by [1], one transmitter sends independent messages to several receivers. The channel under investigation in this paper differs from the original $\mathrm{BC}$ for at least two reasons. First, the receivers are allowed to cooperate in order to enhance the overall network performance. Second, the users want to decode the same message. We will refer to this situation as the cooperative broadcast channel (CBC) with a single common message. For sake of simplicity a 2-user CBC will be assumed. Note that the considered channel is different from the original relay channel (RC) defined in [2] because each terminal not only acts as a relay but also as a receiver, which means that ultimately, the information message has to be decoded by both terminals. Additionally the cooperation channel between the two receivers is assumed to be bidirectional (versus unidirectional for the RC) and orthogonal to the downlink (DL) channels. Although their sub-optimality, orthogonal channels are often assumed for practical reasons (e.g. it is difficult to implement relay-receivers that receive and transmit at the same time in the same frequency band).

To the author's knowledge the most significant contributions ${ }^{1}$ concerning the situation under investigation are [4][5][6][7][8]. The discrete broadcast channel with a bidirectional conference link and a single common message was originally studied by Draper et al. in [4]. The authors proposed a way of decoding the message in multiple rounds and applied their scheme to the binary erasure channel. This channel has also been analyzed by [5] where the authors essentially proposed achievable rates based on the use of estimate-and-forward (EF) at both receivers and two-round cooperation schemes. The

\footnotetext{
${ }^{1}$ For example the authors note that [3] also addressed the $\mathrm{CBC}$ but did not focus on the common message case.
}

Gaussian counterpart of this channel has been studied in [7]. Showing the optimality of decode-and-forward for a unidirectional cooperation the authors evaluated the exact loss due to orthogonalization of the cooperation channel. For the bidirectional case, the proposed achievable rate is based on a combination of EF and decode-andforward and shown to always outperform the pure EF-based solution (always for the 2-round decoding). The fading case has been partially treated in [8]. The diversity-multiplexing trade-off, achieved by using a "dynamic" version of decode-and-forward, is derived for the unidirectional cooperation case.

While the authors of [5][7][8] addressed situations where only one or two cooperation exchanges (or decoding rounds) are allowed, this paper focuses on the case where the number of exchanges is arbitrary. For erasure channels [4] and [6] have shown that the higher the number of exchanges the better the performance in terms of information rate. However the discrete channel analysis (including erasure channels) does not take into consideration the spectral resources aspect. As it will be seen the point is in fact crucial and accounting for it can lead to markedly different conclusions from [4][6] concerning the optimum number of cooperation exchanges. Additionally [4] and [6] only considered the information rate as a performance criterion whereas other criteria of interest can also be considered. This paper aims precisely at taking into account these two aspects for providing some insights to the following issues. If the total bandwidth is fixed what is the optimal number of cooperation exchanges? What is the most efficient way to cooperate: The symmetric or asymmetric way? For the asymmetric cooperation, which user should start cooperating first? What about these issues if the total bandwidth constraint is relaxed?

In order to provide elements of response to these questions we will use a simple approach. After presenting the used system model (sec. 2), we will evaluate the exact equivalent SNR (signal-to-noise ratio) for each user (sec. 3) in the case where amplify-and-forward (AF) is assumed at both receivers. Based on the choice of different system performance criteria (sec. 4) numerical and simulation analyses will be conducted (sec. 5). Concluding remarks and possible extensions of the present work will be given in section 6 .

\section{SYSTEM MODEL}

As mentioned in the introduction the system under investigation comprises one transmitter (source) and two receivers (destinations). The transmitted signal is denoted by $X$ and subject to a power constraint: $E\left[\left|X^{2}\right|\right] \leq P$. Its bandwidth is denoted by $B_{D L}$. For sake of simplicity $X$ will be assumed to be a scalar quantity (e.g. a complex Gaussian input or QAM symbol) in the whole paper. As we will see this simplifying assumption will not prevent us from capturing the 
main issues related to the channel under investigation. Assuming an AWGN model for the DL channels, the received signals write:

$$
\left\{\begin{array}{l}
Y_{1}=X+Z_{1} \\
Y_{2}=X+Z_{2}
\end{array}\right.
$$

where for all $j \in\{1,2\}, Z_{j} \sim \mathcal{N}\left(0, n_{j} B_{D L}\right)$ and $I\left(Z_{1} ; Z_{2}\right)=0$.

We will assume that orthogonality between the DL and cooperation channels is implemented by frequency division (FD). The bandwidth allocated to the cooperation channel between the two receivers is denoted by $B_{C}$. The cooperation channel is then divided into several sub-channels, each of them having a bandwidth equal to $\Delta B$. The two receivers cooperate by applying the same relaying strategy namely the $\mathrm{AF}$ protocol, which imposes $\Delta B=B_{D L}$. Additionally we will assume zero-delay relaying. In real situations, this can be implemented for instance by re-synchronizing the DL and cooperation signals at each receiver. In this paper two main ways to cooperate are distinguished: The symmetric cooperation (figure 1) and asymmetric cooperation (figure 2).

For the symmetric cooperation the two receivers cooperate as follows. Each receiver (e.g. receiver 1) amplifies and forwards his received DL signal $\left(Y_{I}^{(0)}=Y_{1}\right.$ for receiver 1$)$ to his partner (receiver 2). This is done simultaneously. Then each receiver (e.g. receiver 2) applies a maximum ratio combining to the cooperation signal $\left(Y_{12}^{(1)}=a_{12}^{(1)} Y_{I}^{(0)}+Z_{12}^{(1)}\right)$ and DL signal $\left(Y_{I I}^{(0)}=Y_{2}\right)$. As a further step, each receiver amplifies and forwards his MRC output $\left(Y_{I}^{(1)}\right)$. The latter is then received $\left(Y_{12}^{(2)}=a_{12}^{(2)} Y_{I}^{(1)}+Z_{12}^{(2)}\right)$ and recombined with the previous MRC output $\left(Y_{I I}^{(1)}\right)$ and so on. If one denotes by $K_{s}$ the number of pairs of cooperation exchanges we have

$\Delta B=\mid \begin{array}{cccc}\frac{B}{2 K_{s}+1} & \text { when } B_{D L}+B_{C}=\text { const. } \triangleq B \\ B & \text { when } & B_{D L} & =\text { const. } \triangleq B\end{array}$

The first case $B_{D L}+B_{C}=$ const. corresponds to the situation where the system total bandwidth is fixed, which is generally assumed to compare two systems before implementation. The second case $B_{D L}=$ const. does not lead to fair comparisons in terms of bandwidth since the total bandwidth $B=\left(2 K_{s}+1\right) \Delta B=$ $\left(2 K_{s}+1\right) B_{D L}$ increases with $K_{s}$. In fact it is still of practical interest. For instance consider the case where one wants to assess the benefits of cooperation by coupling two existing communication systems such as a DVB (digital video broadcasting) system and a cellular system. As modifying the DVB system downlink signal bandwidth would be a difficult/an impossible task, the second assumption, which amounts to extending the available bandwidth is more appropriate for comparing a DVB system with its terrestrial cooperation-based counterpart. To denote the signals of interest for a given cooperation round or iteration $i$, with $i \in\left\{1, \ldots, K_{s}\right\}$, we will use the following notations:

$$
\left\{\begin{array}{l}
Y_{I}^{(i)}=\alpha_{I}^{(i)} X+Z_{I}^{(i)} \\
Y_{I I}^{(i)}=\alpha_{I I}^{(i)} X+Z_{I I}^{(i)} \\
Y_{12}^{(i)}=a_{12}^{(i)} Y_{I}^{(i-1)}+Z_{12}^{(i)} \\
Y_{21}^{(i)}=a_{21}^{(i)} Y_{I I}^{(i-1)}+Z_{21}^{(i)}
\end{array}\right.
$$

where $a_{12}^{(i)}, a_{21}^{(i)}$ are AF amplification gains, which are determined by the total cooperation powers available: $P_{12}$ at receiver 1 and $P_{21}$ at receiver 2 .

For the asymmetric case, the cooperation is not simultaneous. Assume that receiver 1 starts relaying. For example, receiver 1 sends an amplified version of his DL signal $\left(Y_{I}^{(0)}=Y_{1}\right)$ to receiver 2. Receiver 2 applies an MRC and amplifies and forwards the MRC output $\left(Y_{I I}^{(1)}\right)$ to receiver 1 . Receiver can now combine his DL signal $\left(Y_{I}^{(0)}=Y_{1}\right)$ and cooperation signal $\left(Y_{21}^{(1)}\right)$ to form the new signal $Y_{I}^{(2)}$. If one denotes by $K_{a}$ the number of cooperation exchanges we have

$$
\Delta B=\mid \begin{array}{cccc}
\frac{B}{K_{a}+1} & \text { when } & B_{D L}+B_{C} & =\text { const. } \triangleq B \\
B & \text { when } & B_{D L} & =\text { const. } \triangleq B
\end{array}
$$

We will keep the same notations for the signals of interest as for the symmetric case but in contrast with the symmetric case combining operations take place at receiver 2 for $i$ odd only and at receiver 1 for $i$ even only (under the assumption that receiver 1 starts relaying).

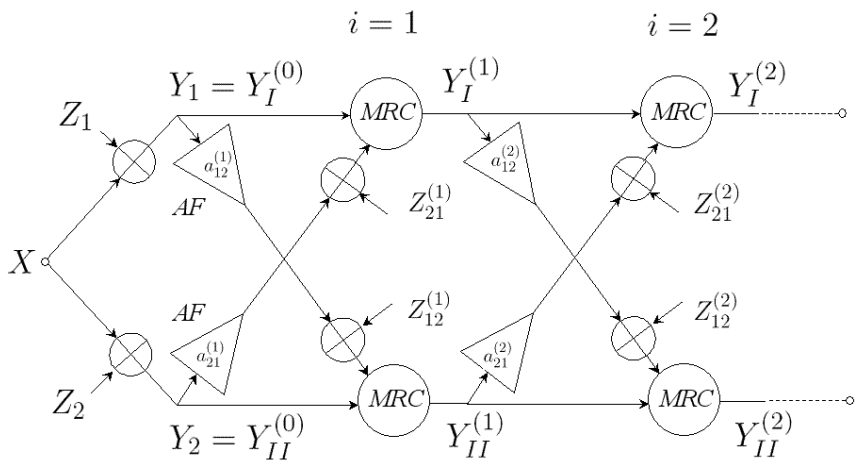

Fig. 1. AF-based symmetric cooperation

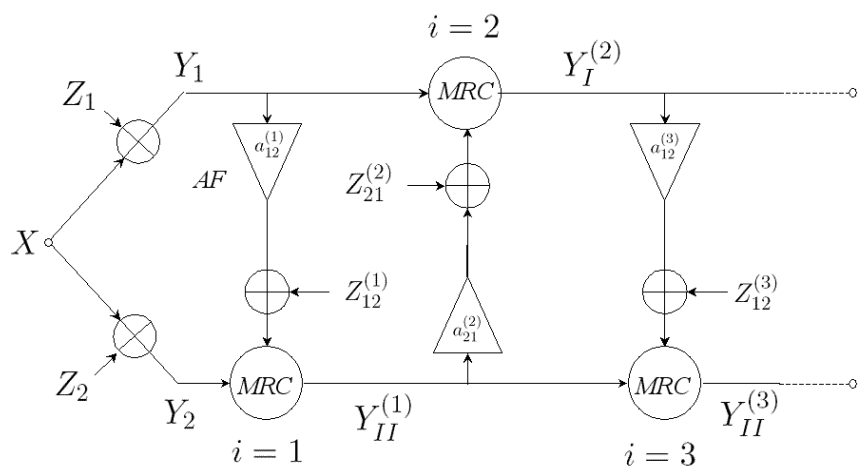

Fig. 2. AF-based asymmetric cooperation

\section{SNR ANALYSIS}

As the DL and cooperation signals are AWGN signals using an MRC for combining these signals is the best choice (in terms of mutual information, of raw BER at the combiner output, etc.). In this section we want to determine the equivalent SNR at the MRC output and use this knowledge to evaluate different performance criteria. It turns out that it is not possible, in general, to express the equivalent SNR as a function of the sole channel parameters $\left(P, P_{12}, n_{1}, \ldots\right)$. In fact the equivalent SNR has to be determined through a recursive formula. The purpose of the following theorem (which proof does not require any specific mathematical tool) is precisely to provide this relationship both for asymmetric and symmetric cooperations. 
Theorem 3.1 (General expression for the equivalent SNRs). Assume that $n_{1}<n_{2}$ and receiver 2 performs the MRC task in the first place if asymmetric cooperation is considered. For iteration $i$ the corresponding weights are denoted by $w_{2}^{(i)}$ (weighting the MRC output at iteration $i-1$ ) and $w_{12}^{(i)}$ (weighting the cooperation signal). For receiver 1 the weights are denoted by $w_{1}^{(i)}, w_{21}^{(i)}$. Denote by $Y_{I}^{(i)}=\alpha_{I}^{(i)} X+Z_{I}^{(i)}$ (resp. $\left.Y_{I I}^{(i)}=\alpha_{I I}^{(i)} X+Z_{I I}^{(i)}\right)$ the signal at MRC output for receiver 1 (resp. receiver 2) and iteration $i$, with $Z_{I}^{(i)} \sim \mathcal{N}\left(0, N_{I}^{(i)}\right)\left(\right.$ resp. $\left.Z_{I I}^{(i)} \sim \mathcal{N}\left(0, N_{I I}^{(i)}\right)\right)$. Let $\rho_{I}^{(i)}\left(\right.$ resp. $\left.\rho_{I I}^{(i)}\right)$ be the signal-to-noise ratio associated with the signal $Y_{I}^{(i)}$ (resp. $\left.Y_{I I}^{(i)}\right)$. The SNRs $\rho_{I}^{(i)} \triangleq \frac{\mathcal{S}_{I}^{(i)}}{\mathcal{T}_{I}^{(i)}}$ and $\rho_{I I}^{(i)} \triangleq \frac{\mathcal{S}_{I I}^{(i)}}{\mathcal{T}_{I I}^{(i)}}$ can be determined recursively as follows:

$$
\begin{aligned}
\mathcal{S}_{I I}^{(i)}= & \alpha_{I}^{(i-1)} \alpha_{I I}^{(i-1)}\left(e^{(i-1)}+e^{(i-1), *}\right) \rho_{I}^{(i-1)} \rho_{I I}^{(i-1)} \rho_{12} \\
& -\left(\alpha_{I}^{(i-1)} \alpha_{I I}^{(i-1)}\right)^{2} P\left[\rho_{I I}^{(i-1)}\left(1+\rho_{I}^{(i-1)}\right)\right. \\
& \left.+\rho_{12}\left(\rho_{I}^{(i-1)}+\rho_{I I}^{(i-1)}\right)\right] \\
\mathcal{T}_{I I}^{(i)}= & \frac{e^{(i-1)} e^{(i-1), *} \rho_{I}^{(i-1)} \rho_{I I}^{(i-1)} \rho_{12}}{P} \\
& -\left(\alpha_{I}^{(i-1)} \alpha_{I I}^{(i-1)}\right)^{2} P\left(1+\rho_{12}\right) \\
& -\left(\alpha_{I}^{(i-1)}\right)^{2} N_{I I}^{(i-1)} \rho_{I}^{(i-1)} \rho_{I I}^{(i-1)}
\end{aligned}
$$

where $\rho_{12}=\frac{P_{12}}{n_{12} \Delta B}, k$ is a constant depending on the cooperation scheme (asymmetric or symmetric), (.) ${ }^{*}$ denotes the conjugate, $e^{(0)}=0, N_{I}^{(0)}=N_{1}, N_{I I}^{(0)}=N_{2}, \rho_{I}^{(0)}=P / N_{1}, \rho_{I I}^{(0)}=P / N_{2}$, $\alpha_{I}^{(0)}=\alpha_{I I}^{(0)}=1$. The cooperative channels gains are defined as: $a_{12}^{(i)}=\sqrt{\frac{P_{12}^{(i)}}{\left(\alpha_{I}^{(i-1)}\right)^{2} P+N_{I}^{(i-1)}}}, a_{21}^{(i)}=\sqrt{\frac{P_{21}^{(i)}}{\left(\alpha_{I I}^{(i-1)}\right)^{2} P+N_{I I}^{(i-1)}}}$ and $P_{12}^{(i)}, P_{21}^{(i)}$ are the available cooperation powers per subchannel. For the $S N R \rho_{I}^{(i)}$ do the following changes for the indices: $I \leftrightarrow I I$ and $1 \leftrightarrow 2$.

The expressions of the signals coefficients $\alpha_{I}^{(i)}, \alpha_{I I}^{(i)}$, the per subchannel cooperation powers $P_{12}^{(i)}, P_{21}^{(i)}$ and the equivalent noise powers $N_{I}^{(i)}, N_{I I}^{(i)}$ depend on the cooperation type. Expressing these quantities is the purpose of the following two propositions.

Proposition 3.2 (MRC weights for the symmetric cooperation). For the symmetric cooperation the MRC weights can be shown to be:

$\left\{\begin{aligned} w_{12}^{(i)} & =a_{12}^{(i)} \alpha_{I}^{(i-1)} N_{I I}^{(i-1)}-a_{12}^{(i)} \alpha_{I I}^{(i-1)} e^{(i-1)} \\ w_{2}^{(i)} & =\left[\left(a_{12}^{(i)}\right)^{2} N_{I}^{(i-1)}+N_{12}^{(i)}\right] \alpha_{I I}^{(i-1)}-\left(a_{12}^{(i)}\right)^{2} \alpha_{I}^{(i-1)} e^{(i-1)} \\ w_{21}^{(i)} & =a_{21}^{(i)} \alpha_{I I}^{(i-1)} N_{I}^{(i-1)}-a_{21}^{(i)} \alpha_{I}^{(i-1)} e^{(i-1)} \\ w_{1}^{(i)} & =\left[\left(a_{21}^{(i)}\right)^{2} N_{I I}^{(i-1)}+N_{21}^{(i)}\right] \alpha_{I}^{(i-1)}-\left(a_{21}^{(i)}\right)^{2} \alpha_{I I}^{(i-1)} e^{(i-1)}\end{aligned}\right.$

where

$$
\begin{aligned}
\cdot e^{(i-1) \triangleq E}[ & \left.Z_{I}^{(i-1)} Z_{I I}^{(i-1)}\right] \text { with } \\
e^{(i)}= & w_{12}^{(i)} a_{12}^{(i)} w_{1}^{(i)} N_{I}^{(i-1)}+w_{21}^{(i)} a_{21}^{(i)} w_{2}^{(i)} N_{I I}^{(i-1)} \\
& +\left[w_{1}^{(i)} w_{2}^{(i)}+w_{12}^{(i)} a_{12}^{(i)} w_{21}^{(i)} a_{21}^{(i)}\right] e^{(i-1)}
\end{aligned}
$$

- for all $i \in\left\{1, \ldots, K_{s}\right\}$ the useful signal coefficients are given by

$$
\left\{\begin{array}{l}
\alpha_{I}^{(i)}=w_{21}^{(i)} a_{21}^{(i)} \alpha_{I I}^{(i-1)}+w_{1}^{(i)} \alpha_{I}^{(i-1)} \\
\alpha_{I I}^{(i)}=w_{12}^{(i)} a_{12}^{(i)} \alpha_{I}^{(i-1)}+w_{2}^{(i)} \alpha_{I I}^{(i-1)}
\end{array}\right.
$$

- the cooperation powers per subchannel are for all $i \in\left\{1, \ldots, K_{s}\right\}$ given by

$$
\left\{\begin{array}{l}
P_{12}^{(i)}=\frac{P_{12}}{K_{s}} \\
P_{21}^{(i)}=\frac{P_{21}}{K_{s}}
\end{array}\right.
$$

- the equivalent noise powers $N_{I}^{(i)}, N_{I I}^{(i)}$ are determined through

$$
\left\{\begin{array}{c}
Z_{I}^{(i)}=w_{21}^{(i)} a_{21}^{(i)} Z_{I I}^{(i-1)}+w_{21}^{(i)} Z_{21}^{(i)}+w_{1}^{(i)} Z_{I}^{(i-1)} \\
Z_{I I}^{(i)}=w_{12}^{(i)} a_{12}^{(i)} Z_{I}^{(i-1)}+w_{12}^{(i)} Z_{12}^{(i)}+w_{2}^{(i)} Z_{I I}^{(i-1)}
\end{array}\right.
$$

- for all $i \in\left\{1, \ldots, K_{s}\right\}: N_{12}^{(i)}=n_{12} \Delta B$ and $N_{21}^{(i)}=n_{21} \Delta B$

- the constant $k$ of Theorem 3.1 equals $\frac{2 K_{s}+1}{K_{s}}$.

Proposition 3.3 (MRC weights for the asymmetric cooperation). For the asymmetric cooperation the MRC weights can be shown to coincide with that of Proposition 3.2 where

- $e^{(i-1)} \triangleq E\left[Z_{I}^{(i-1)} Z_{I I}^{(i-1)}\right]$ with

$$
e^{(i)}=\mid \begin{array}{ll}
w_{1}^{(i)} e^{(i-1)}+w_{21}^{(i)} a_{(21)}^{(i)} N_{I I}^{(i-1)} & \text { for } i \text { even } \\
w_{2}^{(i)} e^{(i-1)}+w_{12}^{(i)} a_{(12)}^{(i)} N_{I}^{(i-1)} & \text { for } i \text { odd },
\end{array}
$$

- the useful signal coefficients are given by

$$
\begin{aligned}
& \alpha_{I}^{(i)}=\mid \begin{array}{cc}
w_{21}^{(i)} a_{21}^{(i)} \alpha_{I I}^{(i-1)}+w_{1}^{(i)} \alpha_{I}^{(i-1)} & \text { for } i \text { even } \\
\alpha_{I}^{(i-1)} & \text { for } i \text { odd },
\end{array} \\
& \alpha_{I I}^{(i)}=\mid \begin{array}{cc}
\alpha_{I I}^{(i-1)} & \text { for } i \text { even } \\
w_{12}^{(i)} a_{12}^{(i)} \alpha_{I}^{(i-1)}+w_{2}^{(i)} \alpha_{I I}^{(i-1)} & \text { for } i \text { odd },
\end{array}
\end{aligned}
$$

- the cooperation powers per subchannel are for all $i \in\left\{1, \ldots, K_{a}\right\}$

$$
\begin{aligned}
& P_{12}^{(i)}=\mid \begin{array}{cc}
\frac{2 P_{12}}{K_{a}} & \text { for } K_{a} \text { even } \\
\frac{2 P_{12}}{K_{a}+1} & \text { for } K_{a} \text { odd }
\end{array} \\
& P_{21}^{(i)}=\mid \begin{array}{cc}
\frac{2 P_{21}}{K_{a}} & \text { for } K_{a} \text { even } \\
\frac{2 P_{21}}{K_{a}-1} & \text { for } K_{a} \text { odd },
\end{array}
\end{aligned}
$$

- the equivalent noise powers $N_{I}^{(i)}, N_{I I}^{(i)}$ are determined through

$$
\begin{aligned}
& Z_{I}^{(i)}=\mid \begin{array}{cc}
w_{21}^{(i)} a_{21}^{(i)} Z_{I I}^{(i-1)}+w_{21}^{(i)} Z_{21}^{(i)}+w_{1}^{(i)} Z_{I}^{(i-1)} & \text { i even } \\
Z_{I}^{(i-1)} & i \text { odd },
\end{array} \\
& Z_{I I}^{(i)}=\mid \begin{array}{cc}
Z_{I I}^{(i-1)} & \text { i even } \\
w_{12}^{(i)} a_{12}^{(i)} Z_{I}^{(i-1)}+w_{12}^{(i)} Z_{12}^{(i)}+w_{2}^{(i)} Z_{I I}^{(i-1)} & i \text { odd },
\end{array}
\end{aligned}
$$

- for all $i \in\left\{1, \ldots, K_{a}\right\}: N_{12}^{(i)}=n_{12} \Delta B$ and $N_{21}^{(i)}=n_{21} \Delta B$, the constant $k$ of Theorem 3.1 equals 1 .

These results will be used in section 5 for evaluating different performance criteria and provide insights to the issues mentioned in the introduction of this paper, in particular to the issue of the optimal number of cooperation exchanges, which is equivalent to a bandwidth allocation issue for the AF protocol. 


\section{PROPOSED SYSTEM PERFORMANCE CRITERIA}

In the simulation section we will dedicate most of our attention to the mutual information criterion but also consider other performance criteria presented here. In order to compare the different cooperation schemes one needs to select suited system performance criterion. For instance, if we fix the information rate/spectral efficiency at the transmitter and obtain the pair of BERs $\left(B E R_{1}, B E R_{2}\right)$ for the coding scheme $\mathcal{C}$ and $\left(B E R_{1}^{\prime}, B E R_{2}^{\prime}\right)$ for the coding scheme $\mathcal{C}^{\prime}$ with $B E R_{1}<B E R_{1}^{\prime}$ and $B E R_{2}>B E R_{2}^{\prime}$ one cannot easily conclude. From now on we will denote by $K$ the number of cooperation exchanges with $K$ equals $K_{a}$ or $K_{s}$ depending on the cooperation scheme. For comparing the AF protocol-based cooperation strategies we propose 4 performance criteria (eq. (2)-(5)).

$$
R_{A F}^{(K)}=B_{D L} \min \left\{\log \left(1+\rho_{I}^{(K)}\right), \log \left(1+\rho_{I I}^{(K)}\right)\right\}
$$

where $\rho_{I}^{(K)}, \rho_{I I}^{(K)}$ are the SNRs at the end of the cooperation procedure. One can notice that $R_{A F}^{(K)}$ represents the maximum information rate possible for a reliable transmission achieved by the proposed cooperation schemes and a Gaussian channel input. Following the proof of [10] for the capacity of the FD relay channel with linear relaying one can show that maximizing $R_{A F}^{(K)}$ over $K$, choosing the best cooperation order and using a convexification procedure with respect to the transmit power (the capacity is a concave function of $P$ ), one can obtain the $\mathrm{CBC}$ capacity for the relaying protocol under consideration.

$$
P_{e, \max }^{(K)}=\max \left\{P_{e, I}^{(K)}, P_{e, I I}^{(K)}\right\}
$$

where $P_{e, I}^{(K)}$ and $P_{e, I I}^{(K)}$ are the raw BERs at the MRC outputs at the end of the cooperation procedure. This criterion is useful in a broadcasting system for which one wants every user to have a minimum transmission quality, which requires $P_{e, \max }^{(K)} \leq P_{e, 0}$ where $P_{e, 0}$ is the minimum quality target. Of course our goal is to minimize $P_{e, \max }^{(K)}$ with respect to $K$.

$$
P_{e, s u m}^{(K)}=P_{e, I}^{(K)}+P_{e, I I}^{(K)} .
$$

This criterion gives an image of the average transmission quality of the broadcasting system. The system probability of errors $P_{e, s y s}^{(K)}$, which is defined as the probability that receiver 1 or (inclusive or) receiver 2 makes a decision error. This probability is generally not easy to explicit but can be bounded by using the last two performance indices:

$$
P_{e, \max }^{(K)} \leq P_{e, s y s}^{(K)} \leq P_{e, s u m}^{(K)} .
$$

Note that the capacity of the channel under consideration is precisely defined with respect to the system error probability, which means that communicating at a rate less than the capacity insures the existence of a code such that $P_{e, s y s}^{K_{o p t}} \rightarrow 0$.

\section{SIMULATION RESULTS}

Asymmetric cooperation: Which user should start cooperating first? Here, the total bandwidth is fixed. Figure 3 represents the plane $\left(n_{1}, n_{2}\right)$ with linear scales: $n_{1} \in\left[10^{-2}, 10^{2}\right], n_{2} \in\left[10^{-2}, 10^{2}\right]$ for different ratios

$\frac{P_{12}}{P_{21}} \in\{-30 \mathrm{~dB},-10 \mathrm{~dB}, 0 \mathrm{~dB}, 10 \mathrm{~dB}, 30 \mathrm{~dB}\}$. The different curves delimit the decision regions that allows us to determine the best cooperation order in terms of information rate. When the cooperation powers are equal (i.e. $\frac{P_{12}}{P_{21}}=0 \mathrm{~dB}$ ) and the pair $\left(n_{1}, n_{2}\right)$ is above the line $n_{2}=n_{1}$ receiver 1 has to start first and conversely. If there is an asymmetry in terms of cooperation powers the answer is less trivial. We represented the decision frontiers for the 5 values for the ratio $\frac{P_{12}}{P_{21}}$. We clearly see that both the DL and cooperation SNRs have to be considered to optimize the overall performance since the frontier can be quite different from the the line $n_{2}=n_{1}$. In practice it can happen that the cooperation powers can be quite close. For instance this is the case if the cooperation powers are chosen to be a fraction of the mobile transmit power in a cellular system. The cooperation order is then less critical.

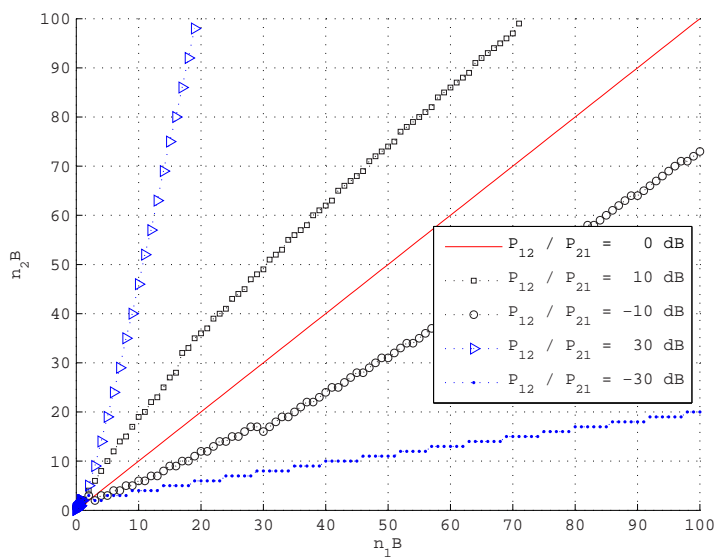

Fig. 3. Decision regions: Who starts cooperating first?

Asymmetric cooperation vs symmetric cooperation. First we assume the total bandwidth to be limited. Figure 4 represents the information rate as the number of cooperation exchanges for the asymmetric $(-*-)$ and symmetric $(-\mathrm{O}-)$ cases for two different scenarios:

$\left(\frac{P}{n_{1} B}, \frac{P}{n_{2} B}, \frac{P_{12}}{n_{12} B}, \frac{P_{21}}{n_{21} B},\right)=(10 \mathrm{~dB}, 0 \mathrm{~dB}, 30 \mathrm{~dB}, 30 \mathrm{~dB})$ and $\left(\frac{P}{n_{1} B}, \frac{P}{n_{2} B}, \frac{P_{12}}{n_{12} B}, \frac{P_{21}}{n_{21} B},\right)=(-1 \mathrm{~dB},-4 \mathrm{~dB}, 30 \mathrm{~dB}, 30 \mathrm{~dB})$. It can be seen that the rate always decreases for $K \geq 2$. This is not surprising since a system with $K>2$ is a special case of the system for which $K=2$. However note that the system with $K=2$ is not a special of the system $K=0$ or $K=1$, which means that cooperating can compensate/overcompensate for the performance loss due do orthogonalizing the DL channel. We also see that the asymmetric system performs better than its symmetric counterpart. We observed from other simulations not presented here that most of the cooperation benefits are captured with one cooperation exchange: In contrast with the discrete CBC with a conference channel [4][5][6] the performance can decrease with $K$. Now we look at two scenarios where the downlink bandwidth is fixed:

$\left(\frac{P}{n_{1} B}, \frac{P}{n_{2} B}, \frac{P_{12}}{n_{12} B}, \frac{P_{21}}{n_{21} B}\right)=(10 \mathrm{~dB}, 10 \mathrm{~dB}, 30 \mathrm{~dB}, 30 \mathrm{~dB})$ and $\left(\frac{P}{n_{1} B}, \frac{P}{n_{2} B}, \frac{P_{12}}{n_{12} B}, \frac{P_{21}}{n_{21} B},\right)=(10 \mathrm{~dB}, 10 \mathrm{~dB}, 6 \mathrm{~dB}, 6 \mathrm{~dB})$. We see that in the high cooperation regime the SIMO bound is rapidly attained that is for $K=2$. When less cooperation powers are available the performance still decreases with $K$. This time this is not due to the orthogonalization loss but to the fact that the cooperation power per exchange decreases in $\sim \frac{1}{K}$ whereas the gain brought by increasing the number of recombinations increases more slowly. Note that now the symmetric system performs better than the asymmetric one because nothing is lost in terms of bandwidth by increasing $K$ (while for the case where the total bandwidth was limited the DL bandwidth was decreasing according to propositions 3.2 and 3.3). 


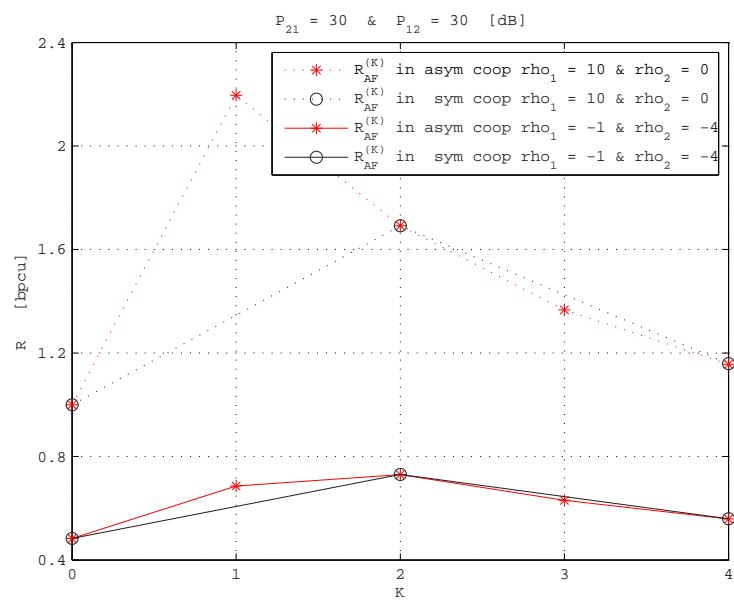

Fig. 4. Achievable rate vs number of cooperation exchanges

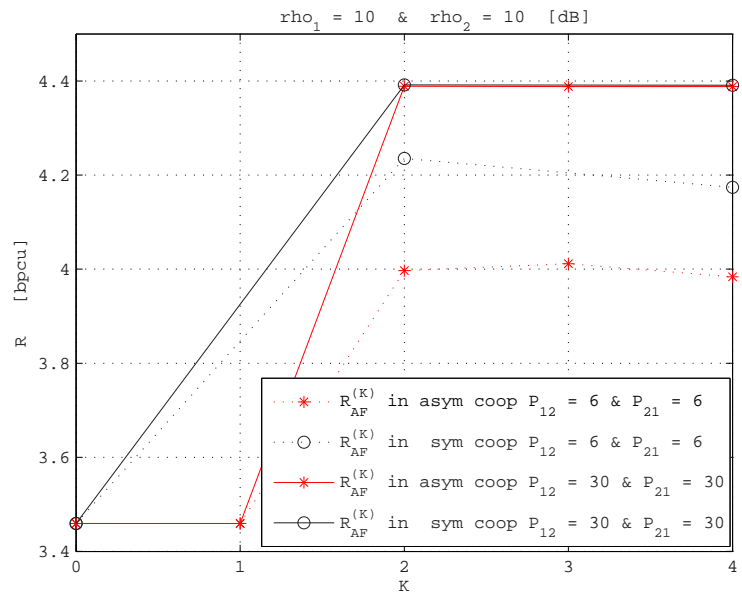

Fig. 5. Achievable rate vs number of cooperation exchanges

Influence of the performance criterion. Simulations such as figure 6 (system BER vs $K$ for a $2^{2(K+1)}$-QAM modulation) have shown that the previous observations are generally confirmed. In the final version a complete commented analysis of this issue will be provided instead of fig. 6 .

\section{CONCLUDING REMARKS}

In this paper we treated the bidirectional $\mathrm{CBC}$ with a single common message when power and spectral resources are limited, which cannot be considered through the discrete approach [4][5][6]. The proposed approach, though very simplified, allowed us to capture the main implementation issues posed by the bidirectional cooperation. In contrast with [4][5][6] we saw that the best performance is generally achieved for one or two cooperation exchanges. The asymmetric cooperation appears as the best choice for a system where the total bandwidth is fixed. The symmetric system performs better than its asymmetric counterpart where only the DL bandwidth is fixed. We insist on the fact that this assumption, although unfair to design

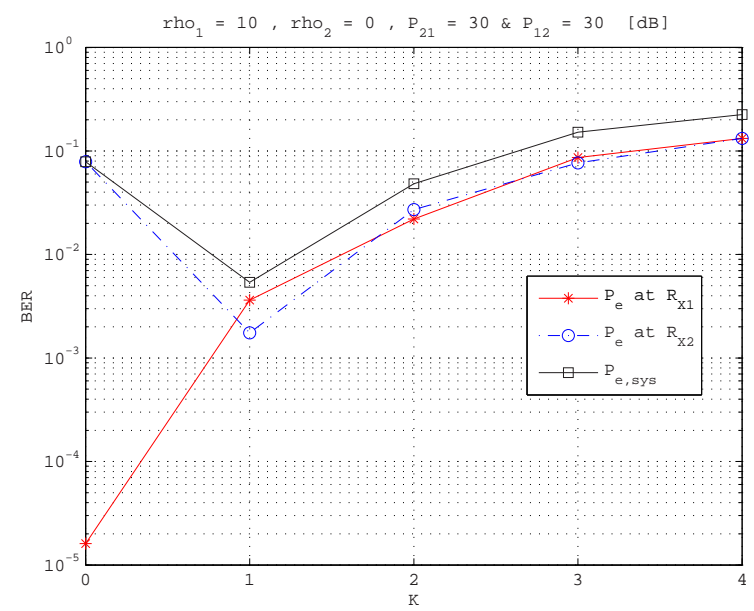

Fig. 6. System BER vs number of cooperation exchanges

a system, still corresponds to real situations (e.g. an existing DVB system coupled with a cellular system). The present work can be extended by considering other relaying protocols (which do not impose the cooperation channel bandwidth to be equal to the DL channel bandwidth) and most importantly fading channels.

\section{REFERENCES}

[1] T. M. Cover, "Broadcast channels", IEEE Trans. Inform. Theory, IT-18(1):2-14, 1972.

[2] T. M. Cover and A. A. El Gamal, "Capacity theorems for the relay channel”, IEEE Trans. Inform. Theory, IT-25(5): 572-584.

[3] Y. Liang and V. V. Veeravalli, "Cooperative relay broadcast channels", To appear in IEEE Trans. on Inform. Theory, March 2007.

[4] S. C. Draper, B. J. Frey, and F. R. Kschischang, "Interactive decoding of a broadcast message", Proc. IEEE Allerton Conf. Commun., Contr., Computing, Oct. 2003.

[5] R. Dabora and S. D. Servetto, "Broadcast channels with cooperating decoders", IEEE Trans. on Inform. Theory, Vol. 52, No. 12, Dec. 2006, pp. 5438-5454.

[6] R. Khalili, S. Lasaulce and P. Duhamel, "Broadcasting a message over erasure channels with cooperating receivers", Proc. IEEE Allerton Conf. Commun., Contr., Computing, Sep. 2006.

[7] S. Lasaulce and A. G. Klein, "Gaussian broadcast channels with cooperating receivers: The single common message case", Proc. IEEE ICASSP, May, 2006.

[8] K. Azarian, H. El Gamal and P. Schniter, "On the achievable diversity-multiplexing tradeoffs in half-duplex cooperative channels", IEEE Trans. Inform. Theory, Vol. 51, No. 12, Dec. 2005.

[9] A. A. El Gamal, "The capacity of a class of broadcast channels", IEEE Trans. Inform. Theory, IT-25, No.2, Mar. 1979, pp. 166169.

[10] A. A. El Gamal, M. Mohseni and S. Zahedi, "Bounds on capacity and minimum energy-per-bit for AWGN relay channels Gamal", IEEE Trans. on Information Theory, Vol. 52, Issue 4, April 2006, pp:1545-1561. 